\title{
Retrieving Transient Magnetic Fields of Ultrarelativistic Laser Plasma via Ejected Electron Polarization
}

\author{
Zheng Gong $\odot{ }^{*}$ Karen Z. Hatsagortsyan $\odot,{ }^{\dagger}$ and Christoph H. Keitel® \\ Max-Planck-Institut für Kernphysik, Saupfercheckweg 1, 69117 Heidelberg, Germany
}

(Received 22 March 2021; revised 2 August 2021; accepted 16 September 2021; published 12 October 2021)

\begin{abstract}
Interaction of an ultrastrong short laser pulse with nonprepolarized near-critical density plasma is investigated in an ultrarelativistic regime, with an emphasis on the radiative spin polarization of ejected electrons. Our particle-in-cell simulations show explicit correlations between the angle resolved electron polarization and the structure and properties of the transient quasistatic plasma magnetic field. While the magnitude of the spin signal is the indicator of the magnetic field strength created by the longitudinal electron current, the asymmetry of electron polarization is found to gauge the islandlike magnetic distribution which emerges due to the transverse current induced by the laser wave front. Our studies demonstrate that the spin degree of freedom of ejected electrons could potentially serve as an efficient tool to retrieve the features of strong plasma fields.
\end{abstract}

DOI: 10.1103/PhysRevLett.127.165002

Magnetic fields play a crucial role in various plasma collective phenomena and nonlinear quantum electrodynamic processes in extreme environments of laboratory and Universe [1-3]. The astrophysical magnetic fields can govern the internal structure of interstellar shocks [4], mediate the radio wave emission nearby neutron stars [5], induce baryon inhomogeneities [6], and catalyze the dark matter formation [7]. Self-generated fields with strength $\sim 10^{4} \mathrm{~T}$ have been produced in high-intensity plasma experiments [8-12], and the guidance of jet flows by laboratory magnetic fields helps interpret the evolution of young stellar objects [13-15]. With recent advancement of ultrastrong laser techniques [16-22] more extreme conditions and larger fields are expected in ultrarelativistic laser plasma interaction [23-29].

Generally, detection of plasma magnetic fields requires an external probe beam, where the field information is imprinted in the velocity space of charged particles [30-35] or the rotated polarization vector of the optical beam [36-39]. However, these conventional methods are inapplicable for scenarios with unprecedented field strength, ultrashort timescale ( $\sim \mathrm{fs})$, and overcritical plasma density [40]. Furthermore, the spin, an intrinsic property of particles, offers a new degree of freedom of information, which is widely utilized in exploring magnetization of solids [41], nucleon structure [42], and phenomena beyond

Published by the American Physical Society under the terms of the Creative Commons Attribution 4.0 International license. Further distribution of this work must maintain attribution to the author(s) and the published article's title, journal citation, and DOI. Open access publication funded by the Max Planck Society. the standard model [43]. In extreme laser fields there is a strong coupling of the electron spin to the laser magnetic field [44-48], which may yield radiative spin polarization (SP) [49-52]; i.e., polarization of electrons due to spin flip during photon emissions. Even though in the oscillating field the electron net SP is suppressed, fast polarization of a lepton beam with laser pulses becomes possible when the symmetry of the monochromatic field is broken, such as in an elliptically polarized, or in two-color laser pulses [53-57]. Because of collective effects, more complex spin dynamics occurs in strong laser field interaction with plasma. Consequently, the question arises if it is possible to employ the spin signal of spontaneously ejected particles from plasma to retrieve information on transient plasma fields.

In this Letter, based on particle-in-cell (PIC) simulations, we investigate the ultrarelativistic dynamics of a short strong pulse interacting with a non-pre-polarized nearcritical density plasma, see Fig. 1. Special attention is devoted to describing the spin dynamics of plasma electrons, being strongly disturbed by the radiative spin flips modulated by the quasistatic plasma magnetic field (QPMF). The latter is commonly transient with a timescale as short as the driving pulse duration while being quasistatic with respect to the fast oscillating laser field. We show that the angle dependent SP of ejected electrons carries signatures of the inhomogeneous QPMF. The signal of SP of ejected electrons can be used to predict the strength of the leading order antisymmetic QPMF created by the longitudinal current. A more detailed analysis reveals that the asymmetry of SP of two outgoing divergent electron bunches characterizes the secondary QPMF, which is induced by a transverse transient current and generally 


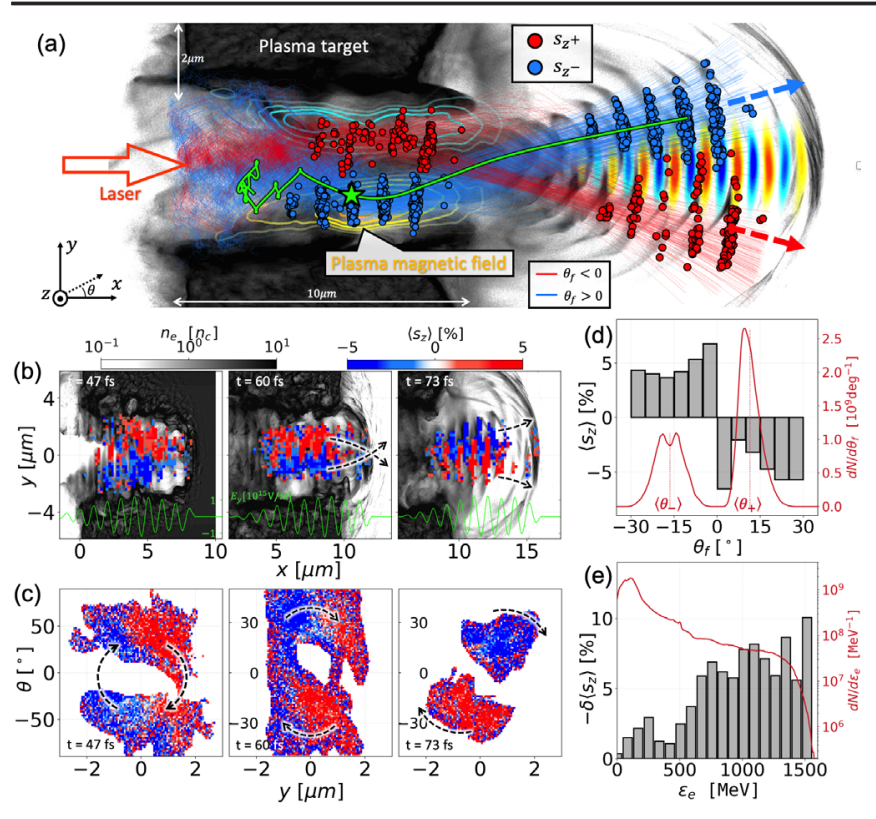

FIG. 1. (a) The interaction scheme: The laser pulse impinges on unpolarized plasma (the electron density is shown in gray shades); accelerated and radiatively polarized electrons due to spin flips form outgoing polarized bunches. The red (blue) dots represent the electrons with spin $s_{z}=1(-1)$ and the lines show their movement tendency. The green line shows a typical electron trajectory with a spin-flip marked by a pentagram. (b) and (c) Snapshots of the electron SP distribution in spatial $(x, y)$ coordinates and transverse $(y, \theta)$ phase space, respectively. The green lines in (b) profile the laser field $E_{y}$ at slice $y=0$. (d) Angular distribution of electron number $d N / d \theta_{f}$ and SP $\left\langle s_{z}\right\rangle$. (e) $\delta\left\langle s_{z}\right\rangle$ and $d N / d \varepsilon_{e}$ vs electron energy $\varepsilon_{e}$. All parameters are indicated in the text.

neglected in previous studies [58-62]. The sum of these two part QPMFs gives rise to a nonlinear islandlike magnetic structure [see Figs. 2(a) and 2(b)]. Our results demonstrate that the spin degree of freedom of ejected electrons from ultrarelativistic plasmas can be employed, in principle, as a tool to retrieve information on the QPMF structure and properties.

In 2D PIC simulations, a near-critical density target is irradiated by a linearly polarized pulse (with the transverse electric field along $y$ ). Our main example adopts a peak intensity of $1.7 \times 10^{23} \mathrm{~W} / \mathrm{cm}^{2}$, equivalent to the normalized field amplitude $a_{0}=350$ given the laser wavelength $\lambda_{0}=1 \mu \mathrm{m}$. The pulse has a $2.6 \mu \mathrm{m}$ focal spot size and 18 fs duration (FWHM intensity measure). The target has thickness $l_{0}=10 \mu \mathrm{m}$ and electron (carbon) density $n_{e}=5 n_{c}$ $\left(n_{i}=n_{e} / 6\right)$, where $n_{c}=m_{e} \omega_{0}^{2} / 4 \pi e^{2}$ is the plasma critical density for a laser frequency $\omega_{0}=c k_{0} ; m_{e}(e)$ the electron mass (charge); $c$ the speed of light. The dynamics of spin precession is governed by the Thomas-BargmannMichel-Telegdi equation and spin-dependent photon emissions have been implemented in the EPOCH code [63], see the Supplemental Material [64], which includes Refs. [65-73].
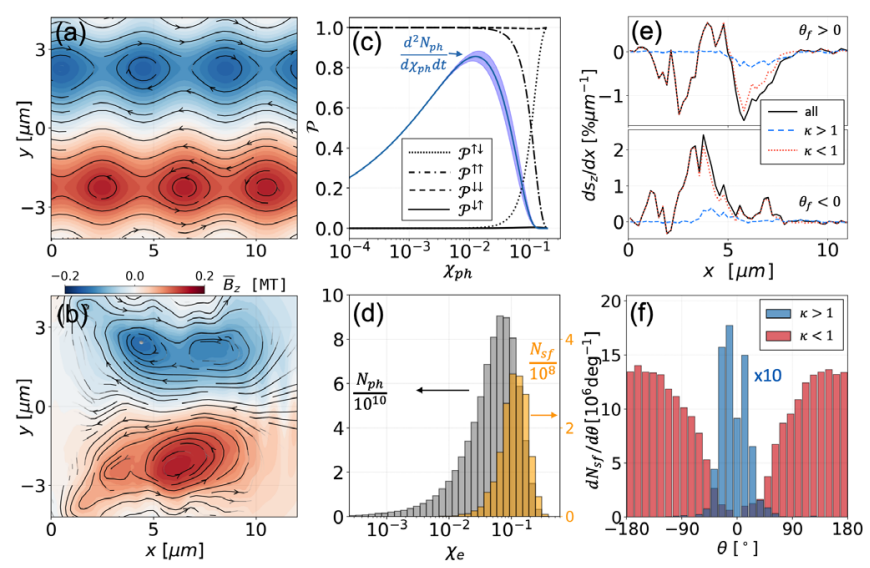

FIG. 2. The $\bar{B}_{z}$ obtained from (a) analytical theory and (b) PIC simulations, where the black arrows denote the direction of electric current $\boldsymbol{j}$. (c) Probabilities $\mathcal{P}$ of electron spin-flip after emitting a photon with $\chi_{\mathrm{ph}}$. The $\uparrow \downarrow$ represents the spin flip from parallel to antiparallel with respect to the magnetic field direction. The blue line profiles the photon emission probability $d^{2} N_{\mathrm{ph}} / d \chi_{\mathrm{ph}} d t$ with a bandwidth accounting for the influence of electron spin. (d) The number distribution of all emitted photons $N_{\text {ph }}$ (gray) and emission associated with spin flips $N_{\text {sf }}$ (yellow). (e) The spatial dependent SP differentiate $d s_{z} / d x$ contributed by the cases of $\kappa \lessgtr 1$ for electrons with final angle $\theta_{f}>0$ and $\theta_{f}<0$, respectively. (f) The angular dependence of spin-flip occurrence, where the result of condition $\kappa>1$ is multiplied by 10 for better visibility.

When the pulse impinges on the target, a fraction of bulk electrons is expelled outwards by the laser ponderomotive force to form a plasma channel [74]. Meanwhile, the peripheral electrons are prone to be injected [75] and subsequently polarized inside the channel due to spin flips during photon emissions, see Fig. 1(b). Since the ion reaction partially compensates the transverse charge separation [76], the quasistatic electric field $\bar{E}_{y}$ is negligible in this scenario. Thus the deflection of the accelerated electrons in transverse direction is predominantly governed by the azimuthal QPMF $\bar{B}_{z}$, which is presumably sustained by the longitudinally forward-moving electron current $j_{x}$. The simulation results in Fig. 1(a) show that the electrons with a positive (negative) final angle $\theta_{f}$ mainly originate from the plasma region of $y<0(y>0)$. As the magnetic field $\bar{B}_{z} \sim-\mu_{0}\left|j_{0}\right| y$ is antisymmetric, created by the nearly uniform current $j_{x} \sim-\left|j_{0}\right|$, the electrons exiting the plasma area with a final angle $\theta_{f}>0$ mostly experience a positive $\bar{B}_{z}$ [see Fig. 1(a)] and vice versa. This leads to oppositely SP ejected electron bunches: $\left\langle s_{z}\right\rangle<0\left(\left\langle s_{z}\right\rangle>0\right)$ for the electron bunch of $\theta_{f}>0\left(\theta_{f}<0\right)$. The spatial evolution of SP in Fig. 1(b) manifests that two groups of electrons are first polarized and confined inside the channel, and then intersect with each other towards the opposite transverse direction. This procedure is also unveiled by the evolution of SP $\left\langle s_{z}\right\rangle$ in the transverse phase space $(y, \theta)$ in Fig. 1(c), 
where $\theta=\arctan \left(p_{y} / p_{x}\right)$ denotes the direction of electron momentum. The clockwise rotation of $\left\langle s_{z}\right\rangle$ indicates that the QPMF not only generates spatial dependent SP but also deflects the electrons to form an angle-dependent polarization distribution of ejected electrons. In Fig. 1(d), asymmetry exists for both electron SP and number angular distributions. Specifically, the averaged SP (final angle) with a positive $\theta_{f}$ is $\left\langle s_{+}\right\rangle \approx-3.3 \%\left(\left\langle\theta_{+}\right\rangle \approx 11.4^{\circ}\right)$, whereas $\left\langle s_{-}\right\rangle \approx 4.0 \%\left(\left\langle\theta_{-}\right\rangle \approx-16.5^{\circ}\right)$ for $\theta_{f}<0$. The magnitude of the SP signal is characterized by the parameter $\delta\left\langle s_{z}\right\rangle \equiv$ $\left\langle s_{+}\right\rangle-\left\langle s_{-}\right\rangle$. According to Fig. 1(e), SP is insignificant for low-energy electrons because of damped radiative spin flips. Therefore, the criterion of $\varepsilon_{e}>4 a_{0} m_{e} c^{2}$ is adopted here to filter out the low-energy noise. To reveal more subtle features of QPMF $\bar{B}_{z}$, we introduce also the spin (angle) asymmetry characteristics via the absolute difference: $\Delta\left\langle s_{z}\right\rangle \equiv\left|\left\langle s_{+}\right\rangle\right|-\left|\left\langle s_{-}\right\rangle\right|$and $\Delta\langle\theta\rangle \equiv\left|\left\langle\theta_{+}\right\rangle\right|-\left|\left\langle\theta_{-}\right\rangle\right|$, which will be discussed below.

The QPMF $\bar{B}_{z}$ is determined by electric currents via $\partial \bar{B}_{z} / \partial y=\mu_{0} j_{x}$ and $\partial \bar{B}_{z} / \partial x=-\mu_{0} j_{y}$ (with the vacuum permeability $\mu_{0}$ ). In general, inside a laser-driven plasma channel, the current is dominated by the longitudinal one $j_{x}$ and the transverse current $j_{y}$ is neglected [58-62]. However, the magnetic field in our simulation shows an irregular structure, with multiple islands associated with the current kinks and vortices, see Fig. 2(b). The latter indicates that the transverse current $j_{y}$ is important in characterizing the exact form of $\bar{B}_{z}$. Let us divide QPMF into two parts $\bar{B}_{z}=\bar{B}_{z, 1}+\bar{B}_{z, 2}$, where the leading part $\bar{B}_{z, 1}$ is induced by $j_{x}$, while the secondary $\bar{B}_{z, 2}$ by $j_{y}: \partial \bar{B}_{z, 1} / \partial y=\mu_{0} j_{x}$ and $\partial \bar{B}_{z, 2} / \partial x=-\mu_{0} j_{y}$. The leading part $\bar{B}_{z, 1} \sim-\mu_{0}|e| n_{e} c y$ with antisymmetric feature $\bar{B}_{z, 1}(-y)=-\bar{B}_{z, 1}(y)$ is ubiquitously utilized in previous studies [58-62]. Now, we focus on the secondary $\bar{B}_{z, 2}$. Considering the electron velocity $v_{y}=$ $p_{y} /\left(\gamma m_{e} c\right)$ and momentum $p_{y} \sim A_{y}=a_{0} \cos \left(\xi+\phi_{0}\right)$, where $\xi=\omega_{0} t-k_{0} x$ and $\phi_{0}$ the carrier envelop phase (CEP), we obtain $j_{y} \approx-|e| \int n_{2} \delta\left(x / v_{g}-t\right) v_{y} d t \approx$ $|e| n_{2} \cos \left[\omega\left(x / v_{g}-x / v_{\mathrm{ph}}\right)+\phi_{0}\right]$. The $\delta\left(t-x / v_{g}\right)$ function indicates that the transverse current is predominantly contributed by the electron density $n_{2} \delta\left(t-x / v_{g}\right)$ piled up at the front edge of the plasma channel nearby the region $x \sim v_{g} t$, where the electron's transverse velocity is significant. Here, $v_{g}\left(v_{\mathrm{ph}}\right)$ is the laser group (phase) velocity in plasma, and the Lorentz factor $\gamma \sim a_{0}$ is assumed. With $\partial \bar{B}_{z, 2} / \partial x=-\mu_{0} j_{y}$, the secondary magnetic field can be estimated:

$$
\bar{B}_{z, 2} \sim-\frac{\mu_{0}|e| n_{2}}{k_{2}} \sin \left(k_{2} x+\phi_{0}\right),
$$

where $k_{2}=k_{0}\left(v_{p h}-v_{g}\right) / v_{g}$. The analytically predicted $\bar{B}_{z}=\bar{B}_{z, 1}+\bar{B}_{z, 2}$ is shown in Fig. 2(a), which agrees qualitatively with the simulated $\bar{B}_{z}$ in Fig. 2(b). The asymmetric periodic islandlike structure of QPMF $\bar{B}_{z}$ stems from the nontrivial current vortex $(\nabla \times \boldsymbol{j})_{x, y} \neq 0$ generated by the transverse current of electrons ploughed away by the laser beam front.

As we are interested in the relation of the electron SP to the magnetic field structure, and considering the polarization attributable to the spin-flip during a photon emission, we analyze the probability of this process $\mathcal{P}\left(\chi_{\mathrm{ph}}\right)$ in Fig. 2(c) for typical parameters of our PIC simulations. Here, the electron with an initial $\gamma_{e}=2000$ normally crosses the uniform magnetic field $B_{0}=10^{4} \mathrm{~T}$, and the electron quantum invariant parameter is $\chi_{e} \sim 0.1$, with $\chi_{e, \mathrm{ph}} \equiv\left(e \hbar / m_{e}^{3} c^{4}\right)\left|F_{\mu \nu} p^{\nu}\right|$ and the momentum $p^{\nu}$ of the electron or photon, respectively. As Fig. 2(c) illustrates, the electron spin flips exclusively take place when emitting an energetic photon with $\chi_{\mathrm{ph}}$ close to $\chi_{e}$, while the photon emission probability is peaked at $\hbar \omega_{c} \sim \chi_{e} \gamma_{e} m_{e} c^{2}$ (at $\left.\chi_{e}<1\right)$, i.e., the peak of the spin-flip process is shifted with respect to the photon emission to higher $\chi_{e}$ 's, see Fig. 2(d). Both the laser magnetic field $B_{l}$ and QPMF $\bar{B}_{z}$ can cause the electron spin flip as $\chi_{e} \approx \gamma_{e} \mid\left[(1-\cos \theta) B_{l}+\right.$ $\left.\bar{B}_{z}\right] \mid / B_{c}$ with the Schwinger limit $B_{c} \approx 4 \times 10^{9} \mathrm{~T}$. We introduce the parameter $\kappa=\mid \bar{B}_{z} /\left[(1-\cos \theta) B_{l} \mid\right]$, defining two regimes, when the electron spin flip is dominated by the plasma $(\kappa>1)$ or by the laser field $(\kappa<1)$. The evolution of SP in Fig. 2(e) demonstrates that the laser field dominated regime $(\kappa<1)$ mostly contributes to the final electron SP. A distinguishable feature between the $\kappa \lessgtr 1$ regimes is the angle $\theta$ of the electron's instantaneous momentum when the spin flip occurs. As the angular dependent spin flip shows in Fig. 2(f), the $\kappa<1$ regime applies at backward emissions, while $\kappa>1$ for forward ones.

The detailed particle tracking further confirms these conclusions. In the $\kappa>1$ regime Figs. 3(a) and 3(b), the position of spin flip with $\kappa>1$ is closely correlated with the spatial distribution of QPMF $\bar{B}_{z}$. The time evolution of $p_{x}$ illustrates that the spin flip happens after the electron starts an efficient acceleration and its velocity aligns longitudinally $\theta \ll 1$, resulting in $(1-\cos \theta) B_{l}<\bar{B}_{z}$. For the laser dominant regime $\kappa<1$, the electron trajectory and momentum evolution [in Figs. 3(c) and 3(d)] demonstrate that the typical spin flip occurs at the electron's temporarily backward motion, when $(1-\cos \theta) \sim 1$ and $B_{l}>\bar{B}_{z}$.

It should be noted that even in the laser dominant regime, the QPMF $\bar{B}_{z}$ is still the key factor for the SP. The reason is that the laser field has oscillating character. Although it can cause spin flips, its net contribution to the final SP is negligible. The laser magnetic field $B_{l}$ acts as a catalyst to enhance the electron spin flips by increasing $\chi_{e}$ and net spin flips contributing to the final SP are still determined by $\bar{B}_{z}$ [64]. We may estimate $s_{z} \approx-\int \bar{B}_{z} /\left|B_{l}\right| \mathcal{A}\left(\chi_{e}\right) d t$ (at $B_{l} \gg \bar{B}_{z}$ ), with $\mathcal{A}\left(\chi_{e}\right)=\left(\sqrt{3} \alpha_{f} m_{e} c^{2} \chi_{e}\right) /\left(h \gamma_{e}\right) \mathcal{A}^{*}\left(\chi_{e}\right)$, 

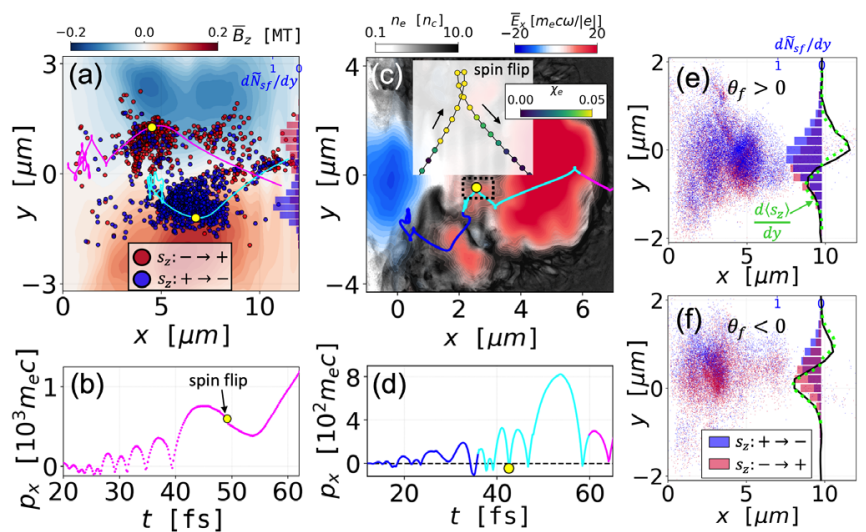

FIG. 3. (a) The red (blue) dots present the electron spin-flip from $s_{z}=-1$ (1) to $1(-1)$ in the plasma field dominant regime $(\kappa>1)$ and the histogram exhibits its dependence on the transverse coordinate $d \tilde{N}_{s f} / d y$. The magenta line refers to a typical electron trajectory and its time evolution of $p_{x}$ is in (b). (c) The typical electron trajectory for the regime $\kappa<1$ and its corresponding momentum evolution in (d). The spin flips of electron trajectories in (a)-(d) are marked by yellow circles. (e) and (f) Spin flips with $\kappa<1$ for electrons with final angle $\theta_{f}>0$ and $\theta_{f}<0$, where the solid black (dashed lime) lines profile the simulated (analytically derived) dependence of net electron SP $s_{z}$ on the coordinate $y$.

and $\mathcal{A}^{*}\left(\chi_{e}\right) \approx 0.18 \chi_{e}$ (at $0.01<\chi_{e}<0.4$ ) [64]. The electrons with final angle $\theta_{f}>0\left(\theta_{f}<0\right)$ are mainly exposed to the QPMF $\bar{B}_{z}>0\left(\bar{B}_{z}<0\right)$ at the region $y<0(y>0)$, and the overall SP with $\theta_{f}>0\left(\theta_{f}<0\right)$ would be $s_{z}<0$ $\left(s_{z}>0\right)$ which are illustrated as the solid black lines in Figs. 3(e) and 3(f).

Thus, we calculate the electron's SP magnitude $\delta\left\langle s_{z}\right\rangle$ being correlated with the leading order QPMF $\bar{B}_{z, 1}$ :

$$
\delta\left\langle s_{z}\right\rangle \sim-\eta \frac{|e|\left|\bar{B}_{z, 1}\right|}{m_{e} \omega_{0}} a_{0}^{2},
$$

where $\gamma_{e} \sim a_{0}$ is used, and $\eta \approx 4 \times 10^{-8}$ accounts for the deviations from the radiative spin evolution. With $\bar{B}_{z, 1} \approx \sqrt{\left(a_{0} / 4 \pi\right)\left(n_{e} / n_{c}\right)}\left(m_{e} \omega_{0} /|e|\right)$, we find the SP scaling $\delta\left\langle s_{z}\right\rangle \propto a_{0}^{5 / 2}$, as well as the relation $\bar{B}_{z, 1} \approx\left[-\left(\delta\left\langle s_{z}\right\rangle / \eta\right)\left(n_{e} / 4 \pi n_{c}\right)^{2}\right]^{1 / 5}$. In Figs. 4(a)-4(c), the analytically predicted scalings of $\delta\left\langle s_{z}\right\rangle$ and $\bar{B}_{z, 1}$ are in good accordance with the 2D simulation results.

Finally, we show how with the help of the SP asymmetry signal $\Delta\left\langle s_{z}\right\rangle$ defined above, the secondary QPMF can be retrieved. In the $\Delta\left\langle s_{z}\right\rangle$ signal the contribution of the $\bar{B}_{z, 1}$ is cancelled, and $\Delta\left\langle s_{z}\right\rangle \approx 2 \int\left(\bar{B}_{z, 2} /\left|B_{l}\right|\right) \mathcal{A}\left(\chi_{e}\right) d t$. Since $\bar{B}_{z, 2} \sim b_{2} \sin \left(k_{2} x+\phi_{0}\right)$ is oscillating along the longitudinal position (along the laser CEP), the overall effect of $\bar{B}_{z, 2}$ imprinted on the signal of $\Delta\left\langle s_{z}\right\rangle$ is oscillating as well. Taking into account the results for $\delta\left\langle s_{z}\right\rangle$ and $\bar{B}_{z, 2}$, we find for the asymmetry signal
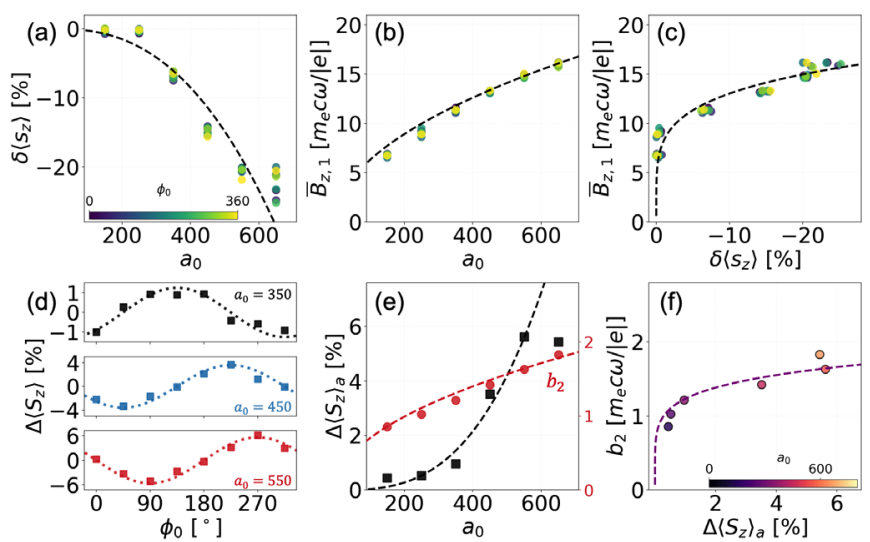

FIG. 4. The dependence of (a) electron SP $\delta\left\langle s_{z}\right\rangle$ and (b) the leading order QPMF $\bar{B}_{z, 1}$ on $a_{0}$. (c) The correlation between $\delta\left\langle s_{z}\right\rangle$ and $\bar{B}_{z, 1}$. In (a)-(c), the dots refer to the simulation results with different laser CEP $\phi_{0}$ while the dashed black line denotes the theory. (d) The dependence of $\Delta\left\langle s_{z}\right\rangle$ on $\phi_{0}$ for different $a_{0}$. (e) The dependence of $\Delta\left\langle s_{z}\right\rangle_{a}$ and $b_{2}$ on $a_{0}$. (f) The correlation between the amplitude of the SP asymmetry $\Delta\left\langle s_{z}\right\rangle_{a}$ and the secondary QPMF $b_{2}$.

$$
\Delta\left\langle s_{z}\right\rangle \sim \delta\left\langle s_{z}\right\rangle \frac{b_{2}}{\left|\bar{B}_{z, 1}\right|} \frac{k_{0}}{k_{2}} \cos \left(k_{2} l_{0}+\phi_{0}\right)
$$

where $b_{2}$ is the amplitude of $\bar{B}_{z, 2}$. The oscillating dependence of $\Delta\left\langle s_{z}\right\rangle$ on the laser CEP $\phi_{0}$ is reproduced by the simulation results in Fig. 4(d). We see that the amplitude of the SP asymmetry signal $\Delta\left\langle s_{z}\right\rangle_{a}$ is directly related to the secondary QPMF $b_{2}: \Delta\left\langle s_{z}\right\rangle_{a} \sim\left|\delta\left\langle s_{z}\right\rangle\right|\left(b_{2} /\left|\bar{B}_{z, 1}\right|\right)\left(k_{0} / k_{2}\right) \sim$ $\eta \sqrt{n_{c} / n_{e}} b_{2} a_{0}^{5 / 2} \quad$ [see Fig. 4(e)], where $k_{0} / k_{2} \sim$ $\left(a_{0} n_{c} / n_{e}\right)^{1 / 2}$ is obtained from simulation results [64]. Moreover, the amplitude of the secondary quasistatic magnetic field $b_{2} \approx 0.03 \sqrt{a_{0}\left(n_{e} / n_{c}\right)}\left(m_{e} \omega_{0} /|e|\right)$ can be estimated through the number conservation between the initial undisturbed plasma and the electrons piled up at the front of the channel edge. Then, the correlation between $\Delta\left\langle s_{z}\right\rangle_{a}$ and $b_{2}$ is established: $b_{2} \sim 0.12\left[\Delta\left\langle s_{z}\right\rangle_{a} / \eta\right]^{1 / 6}$, which is in reasonable agreement with the simulation results [see Fig. 4(f)]. Therefore, the SP signals of $\delta\left\langle s_{z}\right\rangle$ and $\Delta\left\langle s_{z}\right\rangle$ allow us to retrieve the strength of the leading and secondary QPMFs.

In addition, the combination of $\Delta\left\langle s_{z}\right\rangle$ and $\Delta\langle\theta\rangle$, allows us to predict the concrete spatial structure of $\bar{B}_{z}$; see Fig. 5(a). Based on the sign of $\Delta\left\langle s_{z}\right\rangle$ and $\Delta\langle\theta\rangle$, the analytically estimated magnetic island structures agree well with the simulation results, see Ref. [64]. We define the limitations of the presented field retrieval model. First, it is applicable when no more than two QPMF islands exist at $y \lessgtr 0$, with a criterion $l_{0}<l_{\text {island }} \sim 1.7 \lambda_{0}\left(a_{0} n_{c} / n_{e}\right)^{1 / 2}$ [64]. Second, to exclude the influence of depolarization, the ejected electrons should experience a half-period of betatron oscillation inside the channel, with a criterion 


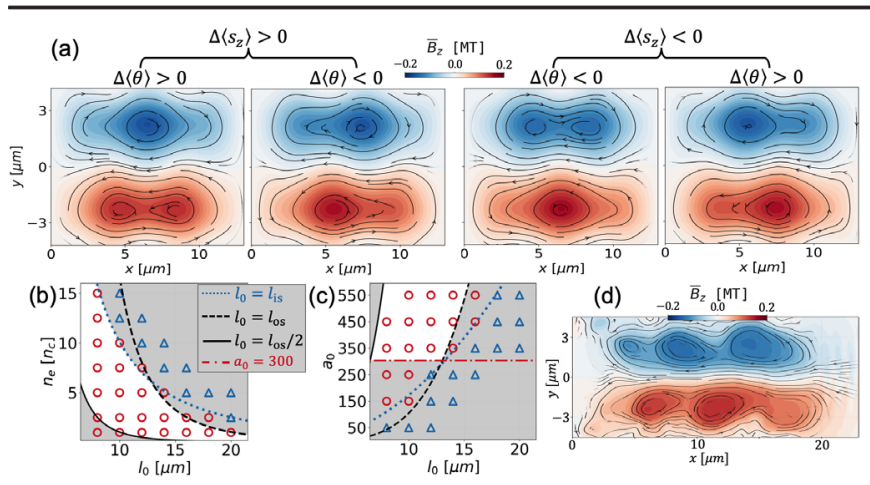

FIG. 5. (a) The $\bar{B}_{z}$ predicted by the model based on the sign of $\Delta\left\langle s_{z}\right\rangle$ and $\Delta\langle\theta\rangle$. The valid range of the model is illustrated in the white region of the parameter space in (b) $a_{0}=350$ and (c) $n_{e}=5 n_{c}$, where the circles (triangles) mark the simulation results of $\bar{B}_{z}$ with no more (more) than two islands on each side of $y=0$. (d) The $\bar{B}_{z}$ obtained from simulation for the case of $a_{0}=350, n_{e}=5 n_{c}$, and $l_{0}=20 \mu \mathrm{m}$.

$0.5 l_{\mathrm{os}}<l_{0}<l_{\mathrm{os}} \sim 5 \lambda_{0}\left(a_{0} n_{c} / n_{e}\right)^{1 / 4} \quad$ [64]. Consequently, the valid range of the model is $0.5 l_{\mathrm{os}}<l_{0}<\min \left\{l_{\mathrm{is}}, l_{\mathrm{os}}\right\}$ shown as the white area in Figs. 5(b) and 5(c). Our method based on the electron radiative polarization will be efficient in the quantum radiation dominated regime at $\alpha_{f} a_{0} \chi_{e} \gtrsim 1$ (approximately at $a_{0} \gtrsim 300$ ) and $\chi_{e} \gtrsim 0.1$ [26], with a SP signal within the precision of the electron polarimetry of $\sim 0.4 \%$ [77]. The requirement might be relieved at alternative setups [29], e.g., in multiple colliding laser pulses [78], where new schemes for the magnetic field retrieval may be needed.

To confirm the robustness of our scheme, we also investigated the role of experimental imperfections and uncertainties, in particular, the asymmetry in the driving laser pulse, and the ramp up and ramp down of the plasma density profile [64]. The simulation results indicate that the presented scheme is robust to moderate imperfections of such practical issues. It should be noted that distinguishing more complex field structures, e.g., the three-island structure like that in Fig. 5(d), could be achievable with modifications of the retrieval method, see an example, in Ref. [64], which, however, needs further exploration.

In conclusion, the ejected electron spin provides a new degree of freedom to extract information on the structure and magnitude of different components of the transient plasma fields. Our results open a new avenue for the electron spin-based plasma diagnostics in extreme conditions, which are prevalent in astrophysical environments and are expected in near future laser facilities.

The original version of code EPOCH adapted here is funded by the UK EPSRC Grants No. EP/G054950/1, No. EP/G056803/1, No. EP/G055165/1, and No. EP/ M022463/1. Z. G. would like to thank A. V. Arefiev, X.-Q. Yan, and J.-X. Li for useful discussions. gong@mpi-hd.mpg.de

'k.hatsagortsyan@mpi-hd.mpg.de

[1] D. Lai, Matter in strong magnetic fields, Rev. Mod. Phys. 73, 629 (2001).

[2] T. Piran, The physics of gamma-ray bursts, Rev. Mod. Phys. 76, 1143 (2005).

[3] R. Ruffini, G. Vereshchagin, and S.-S. Xue, Electronpositron pairs in physics and astrophysics: From heavy nuclei to black holes, Phys. Rep. 487, 1 (2010).

[4] A. Bohdan, M. Pohl, J. Niemiec, P. J. Morris, Y. Matsumoto, T. Amano, M. Hoshino, and A. Sulaiman, Magnetic Field Amplification by the Weibel Instability at Planetary and Astrophysical Shocks with High Mach Number, Phys. Rev. Lett. 126, 095101 (2021).

[5] A. Philippov, A. Timokhin, and A. Spitkovsky, Origin of Pulsar Radio Emission, Phys. Rev. Lett. 124, 245101 (2020).

[6] K. Jedamzik and L. Pogosian, Relieving the Hubble Tension with Primordial Magnetic Fields, Phys. Rev. Lett. 125, 181302 (2020).

[7] J. W. Foster, Y. Kahn, O. Macias, Z. Sun, R. P. Eatough, V. I. Kondratiev, W. M. Peters, C. Weniger, and B. R. Safdi, Green bank and Effelsberg Radio Telescope Searches for Axion Dark Matter Conversion in Neutron Star Magnetospheres, Phys. Rev. Lett. 125, 171301 (2020).

[8] M. Tatarakis, I. Watts, F. Beg, E. Clark, A. Dangor, A. Gopal, M. Haines, P. Norreys, U. Wagner, M.-S. Wei et al., Measuring huge magnetic fields, Nature (London) 415, 280 (2002).

[9] G. Sarri, A. Macchi, C. A. Cecchetti, S. Kar, T. V. Liseykina, X. H. Yang, M. E. Dieckmann, J. Fuchs, M. Galimberti, L. A. Gizzi, R. Jung, I. Kourakis, J. Osterholz, F. Pegoraro, A. P. L. Robinson, L. Romagnani, O. Willi, and M. Borghesi, Dynamics of Self-Generated, Large Amplitude Magnetic Fields following High-Intensity Laser Matter Interaction, Phys. Rev. Lett. 109, 205002 (2012).

[10] W. Schumaker, N. Nakanii, C. McGuffey, C. Zulick, V. Chyvkov, F. Dollar, H. Habara, G. Kalintchenko, A. Maksimchuk, K. A. Tanaka, A. G. R. Thomas, V. Yanovsky, and K. Krushelnick, Ultrafast Electron Radiography of Magnetic Fields in High-Intensity Laser-Solid Interactions, Phys. Rev. Lett. 110, 015003 (2013).

[11] J. Meinecke, H. Doyle, F. Miniati, A. R. Bell, R. Bingham, R. Crowston, R. Drake, M. Fatenejad, M. Koenig, Y. Kuramitsu et al., Turbulent amplification of magnetic fields in laboratory laser-produced shock waves, Nat. Phys. 10, 520 (2014).

[12] C. Huntington, F. Fiuza, J. Ross, A. Zylstra, R. Drake, D. Froula, G. Gregori, N. Kugland, C. Kuranz, M. Levy et al., Observation of magnetic field generation via the Weibel instability in interpenetrating plasma flows, Nat. Phys. 11, 173 (2015).

[13] B. Albertazzi, A. Ciardi, M. Nakatsutsumi, T. Vinci, J. Béard, R. Bonito, J. Billette, M. Borghesi, Z. Burkley, S. Chen et al., Laboratory formation of a scaled protostellar jet by coaligned poloidal magnetic field, Science 346, 325 (2014).

[14] G. Revet, S. N. Chen, R. Bonito, B. Khiar, E. Filippov, C. Argiroffi, D. P. Higginson, S. Orlando, J. Béard, M. Blecher et al., Laboratory unraveling of matter accretion in young stars, Sci. Adv. 3, e1700982 (2017). 
[15] G. Revet, B. Khiar, E. Filippov, C. Argiroffi, J. Béard, R. Bonito, M. Cerchez, S. Chen, T. Gangolf, D. Higginson et al., Laboratory disruption of scaled astrophysical outflows by a misaligned magnetic field, Nat. Commun. 12, 762 (2021).

[16] The Vulcan facility, https://www.clf. stfc.ac.uk/Pages/TheVulcan-10-Petawatt-Project.aspx.

[17] The Extreme Light Infrastructure (ELI), http://www.elilaser.eu/.

[18] ElI-Beamlines, https://www.eli-beams.eu/en/facility/lasers/.

[19] Exawatt Center for Extreme Light Stidies (XCELS), http:// www.xcels.iapras.ru/.

[20] C. N. Danson, C. Haefner, J. Bromage, T. Butcher, J.-C. F. Chanteloup, E. A. Chowdhury, A. Galvanauskas, L. A. Gizzi, J. Hein, D. I. Hillier et al., Petawatt and exawatt class lasers worldwide, High Power Laser Sci. Eng. 7, e54 (2019).

[21] J. W. Yoon, C. Jeon, J. Shin, S. K. Lee, H. W. Lee, I. W. Choi, H. T. Kim, J. H. Sung, and C. H. Nam, Achieving the laser intensity of $5.5 \times 10^{22} \mathrm{w} / \mathrm{cm}^{2}$ with a wavefrontcorrected multi-pw laser, Opt. Express 27, 20412 (2019).

[22] J. W. Yoon, Y. G. Kim, I. W. Choi, J. H. Sung, H. W. Lee, S. K. Lee, and C. H. Nam, Realization of laser intensity over $10^{23} \mathrm{w} / \mathrm{cm}^{2}$, Optica 8, 630 (2021).

[23] G. A. Mourou, T. Tajima, and S. V. Bulanov, Optics in the relativistic regime, Rev. Mod. Phys. 78, 309 (2006).

[24] M. Marklund and P. K. Shukla, Nonlinear collective effects in photon-photon and photon-plasma interactions, Rev. Mod. Phys. 78, 591 (2006).

[25] A. R. Bell and J. G. Kirk, Possibility of Prolific Pair Production with High-Power Lasers, Phys. Rev. Lett. 101, 200403 (2008).

[26] A. Di Piazza, C. Müller, K. Z. Hatsagortsyan, and C. H. Keitel, Extremely high-intensity laser interactions with fundamental quantum systems, Rev. Mod. Phys. 84, 1177 (2012).

[27] S. Bulanov, T. Z. Esirkepov, M. Kando, J. Koga, K. Kondo, and G. Korn, On the problems of relativistic laboratory astrophysics and fundamental physics with super powerful lasers, Plasma Phys. Rep. 41, 1 (2015).

[28] K. Poder et al., Experimental Signatures of the Quantum Nature of Radiation Reaction in the Field of an Ultraintense Laser, Phys. Rev. X 8, 031004 (2018).

[29] J. M. Cole et al., Experimental Evidence of Radiation Reaction in the Collision of a High-Intensity Laser Pulse with a Laser-Wakefield Accelerated Electron Beam, Phys. Rev. X 8, 011020 (2018).

[30] C. K. Li, F. H. Séguin, J. A. Frenje, J. R. Rygg, R. D. Petrasso, R. P. J. Town, P. A. Amendt, S. P. Hatchett, O. L. Landen, A. J. Mackinnon et al., Observation of the Decay Dynamics and Instabilities of Megagauss Field Structures in Laser-Produced Plasmas, Phys. Rev. Lett. 99, 015001 (2007).

[31] L. Willingale, A. G. R. Thomas, P. M. Nilson, M. C. Kaluza, S. Bandyopadhyay, A. E. Dangor, R. G. Evans, P. Fernandes, M. G. Haines, C. Kamperidis, R. J. Kingham, S. Minardi, and M. Notley, Fast Advection of Magnetic Fields by Hot Electrons, Phys. Rev. Lett. 105, 095001 (2010).
[32] A. Macchi, M. Borghesi, and M. Passoni, Ion acceleration by superintense laser-plasma interaction, Rev. Mod. Phys. 85, 751 (2013).

[33] L. Lancia et al., Topology of Megagauss Magnetic Fields and of Heat-Carrying Electrons Produced in a High-Power Laser-Solid Interaction, Phys. Rev. Lett. 113, 235001 (2014).

[34] C. Zhang, J. Hua, Y. Wu, Y. Fang, Y. Ma, T. Zhang, S. Liu, B. Peng, Y. He, C.-K. Huang, K. A. Marsh, W. B. Mori, W. Lu, and C. Joshi, Measurements of the Growth and Saturation of Electron Weibel Instability in Optical-Field Ionized Plasmas, Phys. Rev. Lett. 125, 255001 (2020).

[35] A. F. Bott, P. Tzeferacos, L. Chen, C. A. Palmer, A. Rigby, A. R. Bell, R. Bingham, A. Birkel, C. Graziani, D. H. Froula et al., Time-resolved turbulent dynamo in a laser plasma, Proc. Natl. Acad. Sci. U.S.A. 118, e2015729118 (2021).

[36] M. Tatarakis, A. Gopal, I. Watts, F. Beg, A. Dangor, K. Krushelnick, U. Wagner, P. Norreys, E. Clark, M. Zepf et al., Measurements of ultrastrong magnetic fields during relativistic laser-plasma interactions, Phys. Plasmas 9, 2244 (2002).

[37] M. C. Kaluza, H.-P. Schlenvoigt, S. P. D. Mangles, A. G. R. Thomas, A. E. Dangor, H. Schwoerer, W. B. Mori, Z. Najmudin, and K. M. Krushelnick, Measurement of Magnetic-Field Structures in a Laser-Wakefield Accelerator, Phys. Rev. Lett. 105, 115002 (2010).

[38] A. Buck, M. Nicolai, K. Schmid, C. M. Sears, A. Sävert, J. M. Mikhailova, F. Krausz, M. C. Kaluza, and L. Veisz, Real-time observation of laser-driven electron acceleration, Nat. Phys. 7, 543 (2011).

[39] S. Zhou, Y. Bai, Y. Tian, H. Sun, L. Cao, and J. Liu, SelfOrganized Kilotesla Magnetic-Tube Array in an Expanding Spherical Plasma Irradiated by khz Femtosecond Laser Pulses, Phys. Rev. Lett. 121, 255002 (2018).

[40] T. Wang, T. Toncian, M. Wei, and A. Arefiev, Structured targets for detection of megatesla-level magnetic fields through Faraday rotation of XFEL beams, Phys. Plasmas 26, 013105 (2019).

[41] T. Jungwirth, J. Wunderlich, V. Novák, K. Olejník, B. L. Gallagher, R. P. Campion, K. W. Edmonds, A. W. Rushforth, A. J. Ferguson, and P. Němec, Spin-dependent phenomena and device concepts explored in (Ga, Mn) As, Rev. Mod. Phys. 86, 855 (2014).

[42] K. Abe et al. (E143 Collaboration), Precision Measurement of the Deuteron Spin Structure Function $g_{1}^{d}$, Phys. Rev. Lett. 75, 25 (1995).

[43] G. Moortgat-Pick, T. Abe, G. Alexander, B. Ananthanarayan, A. Babich, V. Bharadwaj, D. Barber, A. Bartl, A. Brachmann, S. Chen et al., Polarized positrons and electrons at the linear collider, Phys. Rep. 460, 131 (2008).

[44] M. W. Walser, D. J. Urbach, K. Z. Hatsagortsyan, S. X. Hu, and C. H. Keitel, Spin and radiation in intense laser fields, Phys. Rev. A 65, 043410 (2002).

[45] D. Del Sorbo, D. Seipt, T. G. Blackburn, A. G. R. Thomas, C. D. Murphy, J. G. Kirk, and C. P. Ridgers, Spin polarization of electrons by ultraintense lasers, Phys. Rev. A 96, 043407 (2017).

[46] D. Seipt, D. Del Sorbo, C. P. Ridgers, and A. G. R. Thomas, Theory of radiative electron polarization in strong laser fields, Phys. Rev. A 98, 023417 (2018). 
[47] D. D. Sorbo, D. Seipt, A. G. R. Thomas, and C. P. Ridgers, Electron spin polarization in realistic trajectories around the magnetic node of two counter-propagating, circularly polarized, ultra-intense lasers, Plasma Phys. Controlled Fusion 60, 064003 (2018).

[48] Y.-F. Li, Y.-Y. Chen, W.-M. Wang, and H.-S. Hu, Production of Highly Polarized Positron Beams via Helicity Transfer from Polarized Electrons in a Strong Laser Field, Phys. Rev. Lett. 125, 044802 (2020).

[49] A. A. Sokolov and I. M. Ternov, Synchrotron Radiation (Akademic, Germany, 1968).

[50] V. N. Baier and V. M. Katkov, Radiational polarization of electrons in inhomogeneous magnetic field, Phys. Lett. 24A, 327 (1967).

[51] V. N. Baier, Radiative polarization of electron in storage rings, Sov. Phys. Usp. 14, 695 (1972).

[52] Y. Derbenev and A. M. Kondratenko, Polarization kinematics of particles in storage rings, Sov. Phys. JETP 37, 968 (1973), https://wiki.jlab.org/ciswiki/images/4/4b/E_037_ 06_0968.pdf.

[53] Y.-F. Li, R. Shaisultanov, K. Z. Hatsagortsyan, F. Wan, C. H. Keitel, and J.-X. Li, Ultrarelativistic Electron-Beam Polarization in Single-Shot Interaction with an Ultraintense Laser Pulse, Phys. Rev. Lett. 122, 154801 (2019).

[54] Y.-F. Li, R.-T. Guo, R. Shaisultanov, K. Z. Hatsagortsyan, and J.-X. Li, Electron Polarimetry with Nonlinear Compton Scattering, Phys. Rev. Applied 12, 014047 (2019).

[55] F. Wan, R. Shaisultanov, Y.-F. Li, K. Z. Hatsagortsyan, C. H. Keitel, and J.-X. Li, Ultrarelativistic polarized positron jets via collision of electron and ultraintense laser beams, Phys. Lett. B 800, 135120 (2020).

[56] Y.-Y. Chen, P.-L. He, R. Shaisultanov, K. Z. Hatsagortsyan, and C. H. Keitel, Polarized Positron Beams via Intense TwoColor Laser Pulses, Phys. Rev. Lett. 123, 174801 (2019).

[57] D. Seipt, D. Del Sorbo, C. P. Ridgers, and A. G. R. Thomas, Ultrafast polarization of an electron beam in an intense bichromatic laser field, Phys. Rev. A 100, 061402(R) (2019).

[58] A. Pukhov and J. Meyer-ter Vehn, Relativistic Magnetic Self-Channeling of Light in Near-Critical Plasma: ThreeDimensional Particle-in-Cell Simulation, Phys. Rev. Lett. 76, 3975 (1996).

[59] A. Pukhov, Z.-M. Sheng, and J. Meyer-ter Vehn, Particle acceleration in relativistic laser channels, Phys. Plasmas 6, 2847 (1999).

[60] D. J. Stark, T. Toncian, and A. V. Arefiev, Enhanced Multi$\mathrm{MeV}$ Photon Emission by a Laser-Driven Electron Beam in a Self-Generated Magnetic Field, Phys. Rev. Lett. 116, 185003 (2016).

[61] Z. Gong, F. Mackenroth, T. Wang, X. Q. Yan, T. Toncian, and A. V. Arefiev, Direct laser acceleration of electrons assisted by strong laser-driven azimuthal plasma magnetic fields, Phys. Rev. E 102, 013206 (2020).

[62] A. E. Hussein, A. V. Arefiev, T. Batson, H. Chen, R. Craxton et al., Towards the optimisation of direct laser acceleration, New J. Phys. 23, 023031 (2021).

[63] T. Arber, K. Bennett, C. Brady, A. Lawrence-Douglas, M. Ramsay, N. Sircombe, P. Gillies, R. Evans, H. Schmitz,
A. Bell et al., Contemporary particle-in-cell approach to laser-plasma modelling, Plasma Phys. Controlled Fusion 57, 113001 (2015).

[64] See Supplemental Material at http://link.aps.org/ supplemental/10.1103/PhysRevLett.127.165002 for detailed numerical models, analytical derivation, and extra simulations.

[65] L. H. Thomas, I. The kinematics of an electron with an axis, London, Edinburgh, Dublin Philos. Mag. J. Sci. 3, 1 (1927).

[66] V. Bargmann, L. Michel, and V. Telegdi, Precession of the Polarization of Particles Moving in a Homogeneous Electromagnetic Field, Phys. Rev. Lett. 2, 435 (1959).

[67] R. Duclous, J. G. Kirk, and A. R. Bell, Monte Carlo calculations of pair production in high-intensity laserplasma interactions, Plasma Phys. Controlled Fusion 53, 015009 (2010).

[68] N. Elkina, A. Fedotov, I. Y. Kostyukov, M. Legkov, N. Narozhny, E. Nerush, and H. Ruhl, QED cascades induced by circularly polarized laser fields, Phys. Rev. ST Accel. Beams 14, 054401 (2011).

[69] C. Ridgers, J. G. Kirk, R. Duclous, T. Blackburn, C. Brady, K. Bennett, T. Arber, and A. Bell, Modelling gamma-ray photon emission and pair production in high-intensity lasermatter interactions, J. Comput. Phys. 260, 273 (2014).

[70] A. Gonoskov, S. Bastrakov, E. Efimenko, A. Ilderton, M. Marklund, I. Meyerov, A. Muraviev, A. Sergeev, I. Surmin, and E. Wallin, Extended particle-in-cell schemes for physics in ultrastrong laser fields: Review and developments, Phys. Rev. E 92, 023305 (2015).

[71] W. Ma, L. Song, R. Yang, T. Zhang, Y. Zhao, L. Sun, Y. Ren, D. Liu, L. Liu, J. Shen et al., Directly synthesized strong, highly conducting, transparent single-walled carbon nanotube films, Nano Lett. 7, 2307 (2007).

[72] J. H. Bin, W. J. Ma, H. Y. Wang, M. J. V. Streeter, C. Kreuzer, D. Kiefer, M. Yeung, S. Cousens, P. S. Foster, B. Dromey, X. Q. Yan, R. Ramis, J. Meyer-ter-Vehn, M. Zepf, and J. Schreiber, Ion Acceleration Using Relativistic Pulse Shaping in Near-Critical-Density Plasmas, Phys. Rev. Lett. 115, 064801 (2015).

[73] H. Y. Wang, C. Lin, Z. M. Sheng, B. Liu, S. Zhao, Z. Y. Guo, Y. R. Lu, X. T. He, J. E. Chen, and X. Q. Yan, Laser Shaping of a Relativistic Intense, Short Gaussian Pulse by a Plasma Lens, Phys. Rev. Lett. 107, 265002 (2011).

[74] A. Pukhov, Strong field interaction of laser radiation, Rep. Prog. Phys. 66, 47 (2002).

[75] L. L. Ji, A. Pukhov, I. Y. Kostyukov, B. F. Shen, and K. Akli, Radiation-Reaction Trapping of Electrons in Extreme Laser Fields, Phys. Rev. Lett. 112, 145003 (2014).

[76] O. Jansen, T. Wang, D. J. Stark, E. dHumières, T. Toncian, and A. Arefiev, Leveraging extreme laser-driven magnetic fields for gamma-ray generation and pair production, Plasma Phys. Controlled Fusion 60, 054006 (2018).

[77] A. Narayan et al., Precision Electron-Beam Polarimetry at $1 \mathrm{GeV}$ Using Diamond Microstrip Detectors, Phys. Rev. X 6, 011013 (2016).

[78] P. Zhang, S. Bulanov, D. Seipt, A. Arefiev, and A. Thomas, Relativistic plasma physics in supercritical fields, Phys. Plasmas 27, 050601 (2020). 